\title{
Real-world prognostic factors in autotransplanted multiple myeloma patients with severe renal impairment: study of the Polish Myeloma Study Group
}

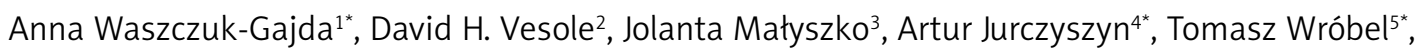

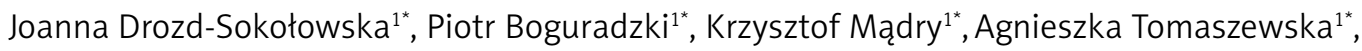
Jarosław Biliński ${ }^{1^{*}}$, Maria Król*1, Longin Niemczyk ${ }^{5}$, Magdalena Olszewska-Szopa ${ }^{{ }^{*}}$, Wieslaw W. Jedrzejczak ${ }^{1^{\star}}$, Grzegorz W. Basak ${ }^{1^{\star}}$

\author{
${ }^{1}$ Department of Haematology, Oncology, and Internal Medicine, Warsaw Medical \\ University, Warsaw, Poland \\ ${ }^{2}$ Myeloma Division, John Theurer Cancer Centre, Hackensack University Medical \\ Centre, NJ, USA \\ ${ }^{3}$ Department of Nephrology, Dialysistherapy, and Internal Diseases, Medical University \\ of Warsaw, Warsaw, Poland \\ ${ }^{4}$ Department of Haematology, Jagiellonian University Medical College, Krakow, Poland \\ ${ }^{5}$ Department and Clinic of Haematology, Blood Neoplasms, and Bone Marrow \\ Transplantation, Wroclaw Medical University, Wroclaw, Poland \\ *Polish Myeloma Study Group
}

Submitted: 18 November 2019

Accepted: 3 February 2020

Arch Med Sci

DOI: https://doi.org/10.5114/aoms.2020.93442

Copyright (c) 2020 Termedia \& Banach

\begin{abstract}
Introduction: The prognostic factors in autotransplanted multiple myeloma (MM) patients with concomitant advanced chronic kidney disease (CKD) are poorly understood, limited, and controversial.

Material and methods: We retrospectively analysed 44 patients with MM and CKD (eGFR $<40 \mathrm{ml} / \mathrm{min}$ ), present both at diagnosis and at autologous stem cell transplantation (ASCT), with no improvement of renal function in-between.

Results: Patients exhibiting deeper paraprotein responses to pre-transplant treatment predicted better response post ASCT (odds ratio $(\mathrm{OR})=11.6, p=$ 0.028 ) and longer progression-free survival (PFS) (hazard ratio $(H R)=0.23$, $p=0.017)$. Higher albumin concentration (per increase of $1 \mathrm{~g} / \mathrm{dl})(\mathrm{HR}=$ $0.41, p=0.03$ ) and melphalan $140 \mathrm{mg} / \mathrm{m}^{2}$ versus higher melphalan doses $(\mathrm{HR}=0.86, p=0.008)$ were associated with longer PFS. Performance status (ECOG $0-1$ versus $\geq 2)(H R=0.28, p=0.0036)$, higher albumin concentration $(H R=0.43, p<0.037)$, and melphalan $140 \mathrm{mg} / \mathrm{m}^{2}$ versus higher melphalan doses $(H R=0.48, p=0.081)$ decreased the risk of death. Three of $32 \mathrm{di}$ alysis-dependent patients became dialysis independent (DID), and 5 of 12 in the DID group had eGFR improvement post ASCT. The median PFS was 2.3 years, which was shorter for DID compared to DD patients (0.7 vs. 3.3 years, respectively). The median overall survival (OS) was 3.6 years, there was no difference in median OS between the groups (4.0 vs. 3.5 years, respectively). Conclusions: Optimal patient selection including good performance status and higher albumin concentration (with every increase of $1 \mathrm{~g} / \mathrm{dl}$ ), chemotherapy-responsive disease pre-ASCT, melphalan dose adjusted to CKD, and intensive post-transplant supportive care are crucial to achieve acceptable results of treatment of MM patients with CKD.
\end{abstract}

Key words: multiple myeloma, advanced chronic kidney disease, autologous stem cell transplantation, dialysis.

\author{
Corresponding author: \\ Anna Waszczuk-Gajda MD, \\ PhD \\ Department \\ of Haematology, \\ Oncology, and \\ Internal Medicine \\ Warsaw Medical University \\ 1a Banacha St \\ 02-097 Warsaw, Poland \\ Phone: +48 225992818 \\ Fax: +48 225991418 \\ E-mail: \\ anna.waszczuk-gajda@wum. \\ edu.pl
}


A. Waszczuk-Gajda, D.H. Vesole, J. Małyszko, A. Jurczyszyn, T. Wróbel, J. Drozd-Sokołowska, P. Boguradzki, K. Mądry, A. Tomaszewska, J. Biliński, M. Król, L. Niemczyk, M. Olszewska-Szopa, W.W. Jedrzejczak, G.W. Basak

\section{Introduction}

Approximately $25-50 \%$ of patients with newly diagnosed multiple myeloma (MM) are diagnosed with renal dysfunction [1, 2]. Successful reversal of renal impairment is multi-faceted and depends on numerous disease and patient characteristics. Renal failure, in general, is associated with shorter survival [3]; however, this is not uniformly true. The aetiology of renal failure in MM patients is multi-factorial, including the direct toxic effect of monoclonal light chains precipitating in the distal collecting renal tubules resulting in tubulointerstitial damage but also dehydration and hypercalcaemia. Moreover, administration of nephrotoxic drugs can also contribute to renal injury. Patients with estimated glomerular filtration rate (eGFR) lower than $30 \mathrm{ml} / \mathrm{min}$ are frequently excluded from clinical trials, and consequently only limited data are available on the safety profile in patients with end-stage renal impairment. The data on the pharmacodynamic and toxicity profile of many drugs in patients with chronic kidney disease (CKD) are also lacking.

Although improvement in renal function, including dialysis independence (DID), in dialysis-dependent (DD) MM patients may be observed after firstline treatment, there remains a group of patients who do not demonstrate improvement in renal function. Patients with extremely reduced eGFR are frequently considered ineligible for autologous stem cell transplantation (ASCT) because of a higher risk of treatment-related toxicities, including death. However, in recent years, in several studies it was found that ASCT may be effective and safe in patients with severe renal impairment [4-9]. Moreover, in some studies it was found that severe renal impairment may be partly reversible in a proportion of patients, especially when ASCT is performed early in the course of the disease [10-12].

In this study, we evaluated the prognostic factors for response, progression-free survival (PFS), and overall survival (OS) in MM patients with severe renal impairment (eGFR lower than $40 \mathrm{ml} /$ min), who underwent ASCT.

\section{Material and methods}

\section{Patients}

The data were analysed of $44 \mathrm{MM}$ patients with advanced CKD at the time of diagnosis and at ASCT (eGFR lower than $40 \mathrm{ml} / \mathrm{min}$ ), who did not show improvement in renal function between diagnosis and ASCT according to International Myeloma Working Group (IMWG) criteria [13]. We used the common definition of renal impairment in $M M$ as eGFR lower than $40 \mathrm{ml} / \mathrm{min}$ or serum creatinine concentration higher than $2 \mathrm{mg} / \mathrm{dl}$ [14]. We excluded patients who were dialysis-inde- pendent at the diagnosis and became DD due to disease progression and those who had an eGFR lower than $40 \mathrm{ml} / \mathrm{min}$ at diagnosis and improved to values greater than $40 \mathrm{ml} / \mathrm{min}$ prior to ASCT.

eGFR was calculated from serum creatinine using the Modification of Diet in Renal Disease (MDRD) equation [15] and the Chronic Kidney Disease Epidemiology (CKD-EPI) Collaboration equation [16]. MDRD is considered a more accurate measure of eGFR than CKD-EPI when eGFR is lower [15]. In DID MM patients, assuming that the baseline eGFR was $<50 \mathrm{ml} / \mathrm{min} / 1.73 \mathrm{~m}^{2}$, renal response was defined according to IMWG 2016 criteria: the complete renal response ( $r C R)$ was defined as best improvement of eGRF $\geq 60 \mathrm{ml}$ min, renal partial response (rPR) as improvement from $<15 \mathrm{ml} / \mathrm{min} / 1.73 \mathrm{~m}^{2}$ to $30-59 \mathrm{ml} / \mathrm{min}$, and renal minor response ( $\mathrm{rMR}$ ) as improvement from $<15 \mathrm{ml} / \mathrm{min}$ to $15-29 \mathrm{ml} / \mathrm{min}$ (best eGFR) or from $15-29 \mathrm{ml} / \mathrm{min}$ to $30-59 \mathrm{ml} / \mathrm{min}$ [13].

All the patients were treated with ASCT between 2004 and 2016 in two Polish transplant centres, and data were collected within the Polish Myeloma Study Group. Records submitted by physicians were evaluated and reviewed for accuracy. The physicians from all over Poland referred eligible patients with dialysis-dependent myeloma to two mentioned centres that have an option of both bone marrow transplantation and nephrology renal replacement therapy in the same institution. The study obtained institutional review board approval of the Medical University of Warsaw for the participating centres.

All the patients had performance status 0-2 according to Eastern Cooperative Oncology Group (ECOG), without significant cardiac, pulmonary, or hepatic dysfunction or active infection. All patients received a high-dose melphalan-containing preparative regimen supported with an infusion of previously collected and cryopreserved autologous peripheral blood stem cells [8]. Melphalan was administered over a 60-minute infusion on day -1 pre ASCT. ASCT was performed on day 0 . A minimum of $2.0 \times 10 \mathrm{CD} 34+$ cells $/ \mathrm{kg}$ were infused. Standard posttransplant supportive care was provided.

\section{Statistical analysis}

Patients characteristics were summarised using the median and interquartile range (IQR) or numbers and percentages for continuous and categorical variables, respectively. The overall response rate was evaluated according to IMWG Uniform Response Criteria [17]. The primary endpoint was OS, calculated from the time of ASCT to the day of the last follow-up or death. Secondary endpoints were: response after transplant according to IMWG [17], PFS calculated from time of ASCT 
to disease progression or death from any cause or the last follow-up, transplant-related mortality (TRM), transplant-related toxicity graded according to Common Toxicity Criteria of Adverse Events 3.0 (CTCAE) [18], and renal improvement according to IMWG [13].

OS and PFS curves were calculated using the Kaplan-Meier method; differences between the subgroups were assessed by log-rank test. Prognostic factors affecting response and PFS/OS after ASCT were evaluated by univariate logistic regression (calculation of odds ratio (OR)) and by univariate Cox's proportional hazards of regression (calculation of hazard ratio (HR)), respectively. Multivariate analysis was not possible to assess due to the low sample size. Results were considered to be statistically significant for $p<0.05$. All calculations were performed using the SAS version 9.4 software package.

Table I. Patients' characteristics $(N=44)\left({ }^{*} N=43\right)$

\begin{tabular}{|c|c|}
\hline Characteristics & $\begin{array}{c}\text { Number (\%) } \\
\text { or median (IQR) }\end{array}$ \\
\hline \multicolumn{2}{|l|}{ At diagnosis: } \\
\hline Age [years] & $56.7(54.7-59.8)$ \\
\hline Age $>60$ year & $10(23)$ \\
\hline Female gender & $23(52)$ \\
\hline Diagnosis before 2012 & $25(57)$ \\
\hline Haemoglobin concentration $[\mathrm{g} / \mathrm{dl}]$ & $9.0(8.2-9.8)$ \\
\hline Albumin concentration $[\mathrm{g} / \mathrm{dl}]$ & $3.5(3.1-4.0)$ \\
\hline $\begin{array}{l}\beta 2-\mathrm{microglobulin} \text { concentration } \\
{[\mathrm{mg} / \mathrm{l}]}\end{array}$ & $32.2(17.0-39.8)$ \\
\hline Creatinine concentration [mg/dl] & $5.6(3.7-8.8)$ \\
\hline GFR CKD-EPI [ml/min] & $8.2(4.8-13.5)$ \\
\hline GFR MDRD [ml/min] & $8.8(5.1-14.3)$ \\
\hline Dialysis dependence & $32(73)$ \\
\hline \multicolumn{2}{|l|}{ Paraprotein type: } \\
\hline $\lg G$ & $21(48)$ \\
\hline $\operatorname{IgA}$ & $4(9)$ \\
\hline $\lg D$ & $2(4.5)$ \\
\hline Light Chain Lambda & $10(22.5)$ \\
\hline Light Chain Kappa & $7(16)$ \\
\hline \multicolumn{2}{|c|}{ Stage according to Durie-Salmon classification: } \\
\hline $1 \mathrm{~B}$ & $1(2)$ \\
\hline $2 \mathrm{~B}$ & $2(4)$ \\
\hline $3 B$ & $41(94)$ \\
\hline Bone lytic change & $44(100)$ \\
\hline \multicolumn{2}{|l|}{ Before ASCT: } \\
\hline \multicolumn{2}{|c|}{ General performance status according to WHO: } \\
\hline 0 & $24(55)$ \\
\hline 1 & $8(18)$ \\
\hline 2 & $12(27)$ \\
\hline
\end{tabular}

\section{Results}

\section{Patients}

All the patients had CKD (32 patients required dialysis) both at diagnosis and ASCT and had no improvement of renal function between diagnosis and ASCT (Table I). Twelve patients had severe CKD but were DID. All patients had ISS (International Staging System) 3 MM [19]. Data on cytogenetic risk groups are missing. The median time from diagnosis to ASCT was 280 days (interquartile range (IQR): 197368). Pre-transplant responses included complete response (CR), very good partial response (VGPR), and partial response (PR) observed in $8(19 \%)$, $9(21 \%)$, and 16 (37\%) of the 33 responding patients, respectively. The 11 (25\%) remaining patients did not achieve at least PR. Melphalan doses ranged from 100 to $200 \mathrm{mg} / \mathrm{m}^{2}: 100 \mathrm{mg} / \mathrm{m}^{2}$ in $11 \%$,

\begin{tabular}{|c|c|}
\hline Characteristics & $\begin{array}{c}\text { Number (\%) } \\
\text { or median (IQR) }\end{array}$ \\
\hline$\geq 1$ comorbidity & $18(41)$ \\
\hline \multicolumn{2}{|l|}{ First line chemotherapy regimens: } \\
\hline $\begin{array}{l}\text { Thalidomide-based } \\
\text { chemotherapy }\end{array}$ & $21(48 \%)$ \\
\hline $\begin{array}{l}\text { VAD (vincristine, Adriamycin, } \\
\text { dexamethasone) }\end{array}$ & $12(27)$ \\
\hline $\begin{array}{l}\text { Bortezomib-based } \\
\text { chemotherapy }\end{array}$ & $8(18)$ \\
\hline $\begin{array}{l}\text { Ctx-Dex (cyclophosphamide, } \\
\text { dexamethasone) }\end{array}$ & $2(4.5)$ \\
\hline ASCT front-line & $1(2)$ \\
\hline $\begin{array}{l}\text { More than one line of induction } \\
\text { therapy }\end{array}$ & $13(30)$ \\
\hline \multicolumn{2}{|l|}{ Response to induction therapy*: } \\
\hline$C R$ & $8(19)$ \\
\hline VGPR & $9(21)$ \\
\hline $\mathrm{PR}$ & $16(37)$ \\
\hline SD & $6(14)$ \\
\hline PD & $4(9)$ \\
\hline ORR $(C R, V G P R, P R)^{\star}$ & $33(77)$ \\
\hline \multicolumn{2}{|l|}{$\begin{array}{l}\text { Conditioning regimen: melphalan } \\
\text { dose: }\end{array}$} \\
\hline $100 \mathrm{mg} / \mathrm{m}^{2}$ & $5(11)$ \\
\hline $140 \mathrm{mg} / \mathrm{m}^{2}$ & $28(64)$ \\
\hline $200 \mathrm{mg} / \mathrm{m}^{2}$ & $11(25)$ \\
\hline \multicolumn{2}{|l|}{ At ASCT: } \\
\hline Creatinine concentration $[\mathrm{mg} / \mathrm{dl}]$ & $5.1(2.2-6.7)$ \\
\hline eGFR CKD-EPI [ml/min] & $10.1(7.2-28.8)$ \\
\hline eGFR MDRD [ml/min] & $10.9(9.0-30.2)$ \\
\hline
\end{tabular}


A. Waszczuk-Gajda, D.H. Vesole, J. Małyszko, A. Jurczyszyn, T. Wróbel, J. Drozd-Sokołowska, P. Boguradzki, K. Mądry, A. Tomaszewska, J. Biliński, M. Król, L. Niemczyk, M. Olszewska-Szopa, W.W. Jedrzejczak, G.W. Basak

$140 \mathrm{mg} / \mathrm{m}^{2}$ in $64 \%$, and $200 \mathrm{mg} / \mathrm{m}^{2}$ in $25 \%$ of the patients (Table I).

\section{Haematological recovery, response, and toxicity}

The median time to neutrophil (ANC $\geq 0.5$ $\left.\times 10^{9} / \mathrm{l}\right)$ and platelet engraftment (>20 × $10^{9} / \mathrm{l}$

Table II. Outcomes of ASCT

\begin{tabular}{|lc|}
\hline Outcome & Results \\
\hline $\begin{array}{l}\text { Time to neutrophil recovery } \\
(\text { Neu } \geq 0.5 \mathrm{G} / \mathrm{l}) \text { [days] }\end{array}$ & $12(11-14)$ \\
\hline $\begin{array}{l}\text { Time to platelet recovery median } \\
(\mathrm{PLT} \geq 20 \mathrm{G} / \mathrm{l}) \text { [days] }\end{array}$ & $11(9-15)$ \\
\hline \begin{tabular}{l} 
Response to ASCT*: \\
\hline CR
\end{tabular} & $11(26 \%)$ \\
\hline VGPR & $8(19 \%)$ \\
\hline PR & $15(35 \%)$ \\
\hline SD & $3(7 \%)$ \\
\hline PD & $6(14 \%)$ \\
\hline $\begin{array}{l}\text { Patients off dialysis - dialysis } \\
\text { independent (N = 32) }\end{array}$ & $3(7 \%)$ \\
\hline Progression-free survival (PFS): & 2.27 \\
\hline Median [years] & 40 \\
\hline 3-year PFS (\%) & 32 \\
\hline 5-year PFS (\%) & 3.6 \\
\hline Overall survival (OS): & 56 \\
\hline Median [years] & 42 \\
\hline 3-year PFS (\%) & \\
\hline 5-year PFS (\%) & \\
\hline
\end{tabular}

Table III. Toxicities $\geq$ grade $3(N=44)$

\begin{tabular}{|c|c|}
\hline Toxicity & $\begin{array}{c}\text { Number (\%) } \\
\text { or median (IQR) }\end{array}$ \\
\hline Mucositis & $20(46)$ \\
\hline Diarrhoea & $11(25)$ \\
\hline Gastrointestinal complications & $2(5)$ \\
\hline $\begin{array}{l}\text { Infections including neutropenic } \\
\text { fever/excluding neutropenic fever }\end{array}$ & $33(75) / 20(43)$ \\
\hline Haemorrhagic complications & $3(7)$ \\
\hline Neurological complications & $3(7)$ \\
\hline 30-days TRM & $1(2)$ \\
\hline \multicolumn{2}{|l|}{ Toxicity management: } \\
\hline Parenteral nutrition & $16(36)$ \\
\hline Usage of opioids & $15(34)$ \\
\hline Platelet concentrate transfusion & $37(77)$ \\
\hline $\begin{array}{l}\text { Number of platelet concentrates } \\
\text { transfused [U] }\end{array}$ & $2.5(1-4)$ \\
\hline $\begin{array}{l}\text { Patients with red blood cell } \\
\text { transfusion (yes vs. no) }\end{array}$ & $34(74)$ \\
\hline Red blood cells transfused [U] & $2(1-4)$ \\
\hline Duration of hospitalisation [days] & $29(20-36)$ \\
\hline
\end{tabular}

without transfusion support was 12 days and 11 days, respectively (Table II). The 30-day TRM was $2 \%$ : one patient died of sepsis (at 17 days post ASCT) (Table III). Of the 43 patients evaluable for response (response was evaluated 3 months after ASCT), 11 (26\%) achieved CR, 8 (19\%) VGPR, and 15 (35\%) PR (Table II). Three (9\%) patients became DID (Table II) (rCR). Of 12 DID multiple myeloma patients with eGFR $<40 \mathrm{ml} / \mathrm{min}, 3$ had GFR improvement fulfilling criteria of renal complete response ( $r C R)$, and 2 had partial renal response (pCR).

The median duration of hospital stay was 29 days (IQR: 20-36 days) (Table III). Parenteral nutrition was required by $16(36 \%)$ patients, and 15 (34\%) patients were treated with opioids. The most common toxicities (all grades) were mucositis (80\%), infections (77\%), and diarrhoea (52\%) (Table III). Platelet transfusion and red blood cell transfusions were needed in 37 (77\%) and 34 (74\%) patients, respectively.

\section{Overall survival and progression-free survival}

With a median follow-up of 3.4 years (confidence interval $(\mathrm{Cl})$ : 0.5-12.7), the median PFS was 2.3 years $(\mathrm{Cl}: 0.8-9.7)$ and median OS was 3.6 years ( $\mathrm{Cl}$ : $0.4-12.7)$ (Figure 1 , Table II). After 5 years, $42 \%$ of patients were alive and $32 \%$ were progression free (Table II).

Survival outcomes of DD patients and DID patients, with severe renal disease, were compared: the PFS was shorter for DID patients compared to DD patients ( 0.7 vs. 3.3 years; due to the small sample size no $p$-value was calculated), and there was no difference in median OS between the two groups (4.0 vs. 3.5 years, respectively) (Table III).

\section{Prognostic factors of response and overall survival}

Overall response (CR, VGPR, or PR) to induction chemotherapy positively predicted response to ASCT $(O R=11.6,95 \% \mathrm{Cl}: 1.31-103, p=0.028)$. Better response to pretransplantation treatment according to IMWG criteria was associated with post-ASCT response.

The presence of lambda light chain monoclonal protein was a negative predictor of VGPR or CR after ASCT (OR = 0.1, 95\% Cl: 0.01-0.82, $p=0.032)$.

Prognostic factors associated with shorter PFS included: presence of proteinuria $(\mathrm{HR}=1.14, \mathrm{Cl}$ : 1.01-1.27, $p<0.029)$ and cardiac complications $(\mathrm{HR}=14.9, \mathrm{Cl}: 2.46-90.2, p<0.003)$, need for parenteral nutrition $(\mathrm{HR}=2.48, \mathrm{Cl} 1.10-5.60, p\rangle$ $0.023)$, and diarrhoea of at least grade $3(\mathrm{HR}=$ 2.32, Cl: $1.01-5.56, p<0.046)$. Predictors of decreased risk of progression were the following: 
A

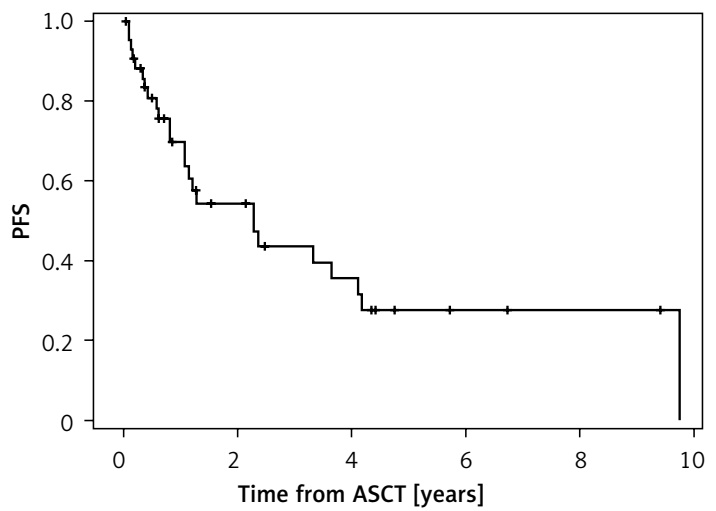

Figure 1. Survival outcomes. A - Progression-free survival (PFS), B - overall survival (OS)
B

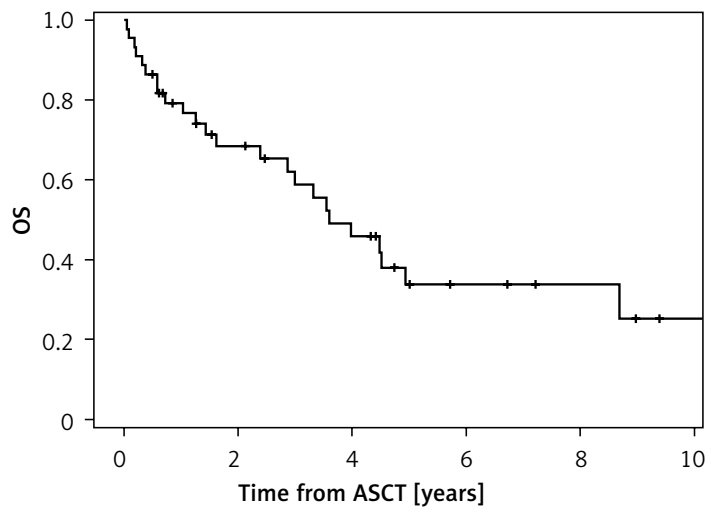

preserved higher albumin concentration (with every increase per $1 \mathrm{~g} / \mathrm{dl})(\mathrm{HR}=0.41, \mathrm{Cl}: 0.18-0.93$, $p<0.03)$, melphalan dose of $140 \mathrm{mg} / \mathrm{m}^{2}$ versus other ( $\mathrm{HR}=0.86, \mathrm{Cl}: 0.15-0.75, p<0.008)$, and achieving $C R$, VGPR, or PR after induction chemotherapy $(\mathrm{HR}=0.23, \mathrm{Cl}: 0.07-0.76, p<0.017)$.

Similarly, higher albumin concentration (with every increase per $1 \mathrm{~g} / \mathrm{dl})(\mathrm{HR}=0.43, \mathrm{Cl}: 0.19-1.0$, $p<0.037$ ) and melphalan dose $140 \mathrm{mg} / \mathrm{m}^{2}$ versus higher melphalan doses $(\mathrm{HR}=0.48, p=0.081)$ were associated with better OS. ECOG performance status of 2 versus 0 and 1 was the only pre-ASCT characteristic negatively affecting OS (OR = $3.595 \% \mathrm{Cl}: 1.51-8.28, p=0.004)$. Shorter OS was associated with requirement for parenteral nutrition post ASCT $(\mathrm{OR}=3.3,95 \% \mathrm{Cl}: 1.44-7.72$, $p=0.005)$, use of narcotic analgesics (OR $=2.7$, $95 \% \mathrm{Cl}: 1.21-6.26, p=0.016)$, grade 3 haemorrhagic complications $(\mathrm{OR}=3.7,95 \% \mathrm{Cl}: 1.07-13.1$, $p=0.039)$, and cardiac complications ( $\mathrm{OR}=15.7$, 95\% Cl: 3.07-80.8, $p=0.001$ ).

The median follow-up time was 3.4 years. Detailed evaluation of prognostic factors affecting overall response rate (ORR), PFS, and OS after ASCT are shown in Table IV.

\section{Discussion}

Renal insufficiency in $M M$ is known to carry poor prognosis [20], and achievement of normal renal function is not a realistic expectation for the majority of patients with DD CKD, in whom renal function did not improve after the first-line treatment $[1,3-6,9]$. Renal insufficiency is one of four myeloma-defining events (MDEs), in addition to anaemia, hypercalcaemia, and bone lesions, as the criteria for initiating MM systemic therapy [17, 21].

However, the treatment options for $M M$ patients with renal failure have improved recently due to the availability of novel agents, which may be safely administered in moderate and end-stage renal insufficiency [22]. For patients with newly diagnosed MM and concomitant renal impairment, proteasome inhibitors (e.g. bortezomib, carfilzomib, ixazomib) based induction is often associated with improvement in renal outcomes [22, 23].

Our group is not large but these are patients from the whole of Poland; the number of patients is also comparable to other studies regarding ASCT in MM patients with CKD $[1,4,12]$. In our series of 44 MM patients with advanced CKD, the frequencies of overall response $\geq$ VGPR were similar before and after ASCT (40\% vs. 45\%, respectively). We can hypothesise that ASCT in the majority of cases consolidated the responses achieved before ASCT, and at least the responses did not worsen. Overall response to induction chemotherapy positively predicted response to ASCT. Attal et al. [24] reported that, in randomly selected MM patients for the treatment with and without ASCT, after four cycles of induction chemotherapy in both groups, an improved response was observed in the transplant setting [25]. However, there are no randomised trials comparing conventional chemotherapy to high-dose therapy and ASCT in MM patients with severe renal impairment, including DD MM patients, so we cannot estimate an absolute benefit of ASCT in DD MM patients regarding response, PFS, and OS. Augeul-Meunier et al. reported response to ASCT of at least VGPR in $58 \%$ of patients and in $96 \%$ of patients with bortezomib-based induction [12]. In contrast to the Augeul-Meunier et al. study, in which $56 \%$ of patients were treated with bortezomib-based induction, only $18 \%$ of our patients received frontline bortezomib. In Poland, bortezomib frontline treatment of patients with severe CKD has only been reimbursed by the National Health Fund since 2012 for transplant-ineligible and since 2015 for transplant-eligible patients (study duration 2004-2016).

It is controversial whether melphalan pharmacokinetics differ significantly in patients with and without renal insufficiency $[26,27]$, but if we assume that there is altered pharmacokinetics of melphalan, especially in $M M$ patients with renal 
A. Waszczuk-Gajda, D.H. Vesole, J. Małyszko, A. Jurczyszyn, T. Wróbel, J. Drozd-Sokołowska, P. Boguradzki, K. Mądry, A. Tomaszewska, J. Biliński, M. Król, L. Niemczyk, M. Olszewska-Szopa, W.W. Jedrzejczak, G.W. Basak

Table IV. Univariate analysis of factors affecting ability to achieve complete response or very good partial response and overall survival after auto-SCT in patients with severe renal insufficiency

\begin{tabular}{|c|c|c|c|c|c|c|c|c|c|}
\hline \multirow[t]{2}{*}{ Characteristics } & \multicolumn{3}{|c|}{$\begin{array}{l}\text { Predictors of CR + VGPR } \\
\text { (logistic regression) }\end{array}$} & \multicolumn{3}{|c|}{$\begin{array}{l}\text { Predictors of PFS (propor- } \\
\text { tional hazard regression) }\end{array}$} & \multicolumn{3}{|c|}{$\begin{array}{l}\text { Predictors of OS (proportion- } \\
\text { al hazards regression) }\end{array}$} \\
\hline & OR & $95 \% \mathrm{Cl}$ & $P$-value & OR & $95 \% \mathrm{Cl}$ & $P$-value & OR & $95 \% \mathrm{Cl}$ & $P$-value \\
\hline Age at diagnosis* & 1.02 & $0.92-1.12$ & 0.771 & 0.99 & $0.93-1.07$ & 0.45 & 1.02 & $0.95-1.09$ & 0.583 \\
\hline $\begin{array}{l}\text { Age }(\text { diagnosis }) \geq 60 \\
\text { years vs. }<60 \text { years }\end{array}$ & 1.36 & $0.33-5.61$ & 0.673 & 1.4 & $0.58-3.39$ & 0.45 & 1.29 & $0.53-3.12$ & 0.567 \\
\hline Sex (woman vs. man) ${ }^{\star *}$ & 1.11 & $0.33-3.71$ & 0.864 & 1.35 & $0.59-3.07$ & 0.47 & 1.47 & $0.65-3.31$ & 0.356 \\
\hline $\begin{array}{l}\text { Diagnosis before } 2012 \\
\text { vs. after 2012**}\end{array}$ & 0.79 & $0.24-2.67$ & 0.709 & 1.5 & $0.62-3.66$ & 0.82 & 1.38 & $0.53-3.58$ & 0.514 \\
\hline ECOG 2 vs. ECOG > $2^{* *}$ & 0.46 & $0.1-2.07$ & 0.309 & 1.57 & $0.61-3.97$ & 0.35 & 0.28 & $0.12-0.66$ & 0.0036 \\
\hline Albumin concentration * & 0.79 & $0.3-2.08$ & 0.627 & 0.41 & $0.18-0.93$ & 0.03 & 0.43 & $0.19-1.0$ & 0.037 \\
\hline $\begin{array}{l}\beta 2 \text {-microglobuline } \\
\text { concentration* }\end{array}$ & 0.99 & $0.95-1.02$ & 0.523 & 1 & $0.95-1.05$ & 0.97 & 0.99 & $0.96-1.03$ & 0.79 \\
\hline Calcium concentration* & 2.91 & $0.33-25.6$ & 0.336 & 0.76 & $0.20-2.72$ & 0.67 & 0.55 & $0.13-2.25$ & 0.405 \\
\hline $\mathrm{Hb}$ concentration* & 1.00 & $0.72-1.38$ & 0.982 & 0.92 & $0.67-1.27$ & 0.62 & 1.043 & $0.74-1.48$ & 0.813 \\
\hline $\begin{array}{l}\text { Plasma cell count in } \\
\text { bone marrow } \geq 60 \% \text { vs. } \\
60 \%^{* *}\end{array}$ & 0.99 & $0.97-1.01$ & 0.434 & 1.02 & $0.99-1.04$ & 0.07 & 1.01 & $0.99-1.03$ & 0.170 \\
\hline $\begin{array}{l}\text { Creatinine concentration } \\
\text { at diagnosis* }\end{array}$ & 0.86 & $0.72-1.04$ & 0.13 & 0.98 & $0.85-1.12$ & 0.71 & 1.02 & $0.91-1.2$ & 0.720 \\
\hline $\begin{array}{l}\text { Creatinine concentration } \\
\text { at auto-SCT* }\end{array}$ & 0.86 & $0.69-1.08$ & 0.194 & 0.89 & $0.77-1.04$ & 0.15 & 0.95 & $0.83-1.1$ & 0.507 \\
\hline $\begin{array}{l}\mathrm{CD} 34(+) \geq 4 \geq 10 \mathrm{mln} / \mathrm{kg} \\
\text { body weight vs. }<4 \times 10 \\
\mathrm{mln} / \mathrm{kg}^{\star \star}\end{array}$ & 1.71 & $1.05-2.78$ & 0.031 & 0.76 & $0.56-1.03$ & 0.07 & 0.87 & $0.65-1.15$ & 0.331 \\
\hline IgG concentration* & 1.00 & $1.0-1.0$ & 0.999 & 0.99 & $0.99-1.06$ & 0.18 & 0.99 & $1.00-1.00$ & 0.109 \\
\hline IgA concentration* & 1.00 & $0.99-1.0$ & 0.594 & 1.01 & $0.99-1.01$ & 0.70 & 1,00 & $1.00-1.00$ & 0.954 \\
\hline $\begin{array}{l}\text { Time from diagnosis to } \\
\text { auto-SCT* }\end{array}$ & 1.00 & $1.0-1.0$ & 0.406 & 0.99 & $0.99-1.00$ & 0.07 & 1,00 & $1.00-1.01$ & 0.341 \\
\hline $\begin{array}{l}\text { Proteinuria vs. no } \\
\text { proteinuria** }^{* *}\end{array}$ & 0.95 & $0.84-1.07$ & 0.406 & 1.14 & $1.01-1.27$ & 0.029 & 1.07 & $0.97-1.17$ & 0.192 \\
\hline $\begin{array}{l}\text { Light chains vs. no light } \\
\text { chains }^{* *}\end{array}$ & 1.00 & $1.00-1.00$ & 0.513 & 1.00 & $1.00-1.00$ & 0.57 & 1,00 & $1.00-1.00$ & 0.979 \\
\hline $\begin{array}{l}\text { FLC type lambda vs. } \\
\text { other }^{\star *}\end{array}$ & 0.09 & $0.01-0.82$ & 0.032 & 1.57 & $0.65-3.82$ & 0.32 & 0.94 & $0.35-2.6$ & 0.906 \\
\hline $\begin{array}{l}\text { Lines of treatment } \\
\text { before auto-SCT } 1 \text { line } \\
\text { vs. } \geq 1 \text { line }^{* *}\end{array}$ & 1.75 & $0.47-6.50$ & 0.403 & 0.38 & $0.13-1.11$ & 0.08 & 0.42 & $0.14-1.2$ & 0.115 \\
\hline $\begin{array}{l}\mathrm{MEL}=200 \mathrm{mg} / \mathrm{m}^{2} \text { vs. } \\
\text { other }^{\star \star}\end{array}$ & 1.07 & $0.27-4.25$ & 0.922 & 2.04 & $0.89-4.71$ & 0.09 & 1.57 & $0.67-3.65$ & 0.300 \\
\hline $\begin{array}{l}\mathrm{MEL}=140 \mathrm{mg} / \mathrm{m}^{2} \text { vs. } \\
\text { other }^{\star *}\end{array}$ & 1.03 & $0.3-3.58$ & 0.965 & 0.86 & $0.15-0.75$ & 0.008 & 0.48 & $0.22-1.09$ & 0.081 \\
\hline $\begin{array}{l}\text { Treatment with newer } \\
\text { drugs in the first line vs. } \\
\text { other }\end{array}$ & 0.73 & $0.22-2.44$ & 0.607 & 1.16 & $0.52-2.59$ & 0.71 & 0.69 & $0.31-1.56$ & 0.380 \\
\hline Comorbidities 1 or $>1^{\star *}$ & 0.57 & $0.16-2.01$ & 0.379 & 1.13 & $0.89-1.42$ & 0.32 & 1.09 & $0.86-1.38$ & 0.480 \\
\hline $\begin{array}{l}\text { CR, VGPR or PR after } \\
\text { chemotherapy vs. other } \\
\text { response }\end{array}$ & 14.44 & $3.06-68.2$ & 0.001 & 0.23 & $0.07-0.76$ & 0.017 & 0.52 & $0.19-1.5$ & 0.202 \\
\hline $\begin{array}{l}\text { CR, VGPR after } \\
\text { chemotherapy vs. other } \\
\text { response** }\end{array}$ & 11.57 & $1.31-103$ & 0.028 & 0.67 & $0.27-1.63$ & 0.38 & 0.89 & $0.35-2.3$ & 0.810 \\
\hline
\end{tabular}


Table IV. Cont.

\begin{tabular}{|c|c|c|c|c|c|c|c|c|c|}
\hline \multirow[t]{2}{*}{ Characteristics } & \multicolumn{3}{|c|}{$\begin{array}{l}\text { Predictors of CR + VGPR } \\
\text { (logistic regression) }\end{array}$} & \multicolumn{3}{|c|}{$\begin{array}{l}\text { Predictors of PFS (propor- } \\
\text { tional hazard regression) }\end{array}$} & \multicolumn{3}{|c|}{$\begin{array}{l}\text { Predictors of OS (proportion- } \\
\text { al hazards regression) }\end{array}$} \\
\hline & OR & $95 \% \mathrm{Cl}$ & $P$-value & OR & $95 \% \mathrm{Cl}$ & $P$-value & OR & $95 \% \mathrm{Cl}$ & $P$-value \\
\hline \multicolumn{10}{|c|}{ Post-transplant complications: } \\
\hline $\begin{array}{l}\text { Mucositis grade } 3,4 \\
\text { versus grade } 1,2 \text { or no } \\
\text { mucositis** }\end{array}$ & 0.39 & $0.11-1.37$ & 0.143 & 1.90 & $0.85-4.26$ & 0.12 & 2.73 & $1.15-6.50$ & 0.023 \\
\hline Parenteral nutrition ${ }^{\star *}$ & 0.32 & $0.08-1.23$ & 0.097 & 2.48 & $1.10-5.60$ & 0.023 & 3.33 & $1.44-7.72$ & 0.005 \\
\hline $\begin{array}{l}\text { Opioids use yes vs. } \\
\text { no** }\end{array}$ & 0.92 & $0.26-3.34$ & 0.903 & 1.65 & $0.72-3.78$ & 0.24 & 2.76 & $1.21-6.26$ & 0.016 \\
\hline $\begin{array}{l}\text { Diarrhea grade } 3 \text { vs. } \\
\text { grade } 1,2 \text { or no** }\end{array}$ & 0.2 & $0.04-1.05$ & 0.058 & 2.32 & $1.01-5.56$ & 0.046 & 0.60 & $0.26-1.4$ & 0.233 \\
\hline $\begin{array}{l}\text { Infection of grade } \geq \\
3 \text { vs. complications } \\
\text { grade } 1,2 \text { or no } \\
\text { complications** }^{*}\end{array}$ & 0.93 & $0.24-3.70$ & 0.922 & 0.99 & $0.39-2.50$ & 0.98 & 1.12 & $0.44-2.84$ & 0.810 \\
\hline $\begin{array}{l}\text { Haemorrhagic } \\
\text { complication of grade } 3 \\
\text { vs. no complications** }\end{array}$ & & $\begin{array}{c}<0.001 \\
>999.99\end{array}$ & 0.998 & 1.64 & $0.38-7.06$ & 0.51 & 3.76 & $1.07-13.1$ & 0.039 \\
\hline $\begin{array}{l}\text { Neurological } \\
\text { complication of grade } \\
3 \text { vs. grade } 1,2 \text { or no } \\
\text { complications** }\end{array}$ & 0.61 & $0.05-7.3$ & 0.697 & 0.67 & $0.09-5.04$ & 0.70 & 2.13 & $0.48-9.4$ & 0.317 \\
\hline $\begin{array}{l}\text { Cardiac complication of } \\
\text { grade } 3 \text { vs. grade } 1,2 \text { or } \\
\text { no complications** }\end{array}$ & $* * *$ & & & 14.9 & $2.46-90.2$ & 0.003 & 32.0 & $5.22-196.8$ & 0.0002 \\
\hline $\begin{array}{l}\text { Neutrophil regeneration } \\
\text { time* }\end{array}$ & 1.04 & $0.86-1.25$ & 0.72 & 1.00 & $0.90-1.13$ & 0.88 & 0.47 & $0.93-1.16$ & 0.472 \\
\hline $\begin{array}{l}\text { Platelet regeneration } \\
\text { time* }\end{array}$ & 1.07 & $0.96-1.18$ & 0.239 & 1.00 & $0.94-1.09$ & 0.79 & 1.03 & $0.96-1.10$ & 0.384 \\
\hline $\begin{array}{l}\text { Platelet concentrate } \\
\text { transfusion (number)* }\end{array}$ & 1.08 & $0.94-1.24$ & 0.294 & 0.98 & 0.8721 .096 & 0.70 & 1.03 & $0.95-1.12$ & 0.460 \\
\hline $\begin{array}{l}\text { Platelet concentrate (yes } \\
\text { or no)** }\end{array}$ & 2.24 & $0.38-13.1$ & 0.372 & 1.57 & $0.53-4.72$ & 0.42 & 2.35 & $0.70-7.94$ & 0.170 \\
\hline $\begin{array}{l}\text { Erythrocyte concentrate } \\
\text { transfusion (number)* }\end{array}$ & 1.05 & $0.92-1.19$ & 0.507 & 1.01 & $0.90-1.13$ & 0.88 & 1.06 & $0.96-1.17$ & 0.239 \\
\hline $\begin{array}{l}\text { Erythrocyte concentrate } \\
(\text { yes or no) })^{\star \star}\end{array}$ & 0.74 & $0.18-3.05$ & 0.673 & 1.51 & $0.59-3.87$ & 0.39 & 2.53 & $0.85-7.55$ & 0.096 \\
\hline Hospitalisation duration* & 1.00 & $0.96-1.04$ & 0.832 & 1.01 & $0.98-1.04$ & 0.37 & 1.02 & $0.97-1.07$ & 0.097 \\
\hline
\end{tabular}

failure, it may result in a higher concentration of the drug and higher incidence of clinical toxicity [27]. Melphalan is subject to spontaneous degradation $[28,29]$ and can be altered by a low concentration of albumin [27, 29]. In our study melphalan $140 \mathrm{mg} / \mathrm{m}^{2}$ (MEL140), compared either to $100 \mathrm{mg} / \mathrm{m}^{2}$ (MEL100) or to $200 \mathrm{mg} / \mathrm{m}^{2}$ (MEL200), was associated with better PFS and OS. Mahindra et al. described 69 patients with severe RI ( $<30 \mathrm{ml} / \mathrm{min}$ ); 34 of 35 DD MM patients achieved dialysis independence post-ASCT [30]. Auner et al. showed an advantage of MEL200 in patients transplanted in less than partial response $(H R=$ 0.5, MEL200 versus MEL140), and ASCT in VGPR or CR significantly favoured MEL140 for OS (HR = 2.02) [31]. Impaired renal function did not favour the use of a lower melphalan dose with regards to non-relapse mortality or haematopoietic recovery. The dosing of melphalan in the treatment of MM seems to be crucial $[4,7,11,13,31-35]$. In the consensus statement of the International Myeloma Working Group regarding treatment of $M M$ patients with renal impairment, high-dose melphalan $140 \mathrm{mg} / \mathrm{m}^{2}$ is recommended rather than melphalan $200 \mathrm{mg} / \mathrm{m}^{2}$ [24].

Patients with lambda light chain disease (10 out of 44) had a lower response rate than patients with other immunoglobulin isotypes. Lambda light chains are found to be more toxic to renal tubules and associated with shorter survival in comparison to patients with kappa light chain disease in other studies, as well $[36,37]$. 
In patients with severe renal impairment, factors such as metabolic abnormalities, electrolyte, and fluid shifts may contribute to cardiac complications. Moreover, these patients may also have an increased risk of cardiovascular disease such as coronary heart disease, chronic heart failure, and other cardiovascular complications also without ASCT [38, 39].

With a median OS of 3.6 years and a median PFS of 2.3 years, our results are comparable to those observed in the other reports of $M M$ patients with advanced CKD [4, 5], but they are inferior to those reported by Augeul-Meunier et al. [12], where OS and PFS were 6.3 years and 4.6 years, respectively. In our study the inclusion criteria also included the absence of renal response (any renal response) during the treatment period between diagnosis and ASCT according to IMWG criteria [13]. While in the study of Augeul-Meunier et al. of 26 patients who were DD on diagnosis 23 remained DD at ASCT, we did not include patients who became DID between induction chemotherapy and ASCT or who fulfilled criteria of minor response.

Three patients of our group (9\%) became dialysis independent after ASCT. In the published studies the range of dialysis independence post ASCT varies between $3.4 \%$ and $24 \%[7,10,12$, 37]. Lee et al. suggested that a higher rate of independence from dialysis after ASCT may be related to shorter duration of dialysis before ASCT ( $<6$ months) and recommend ASCT as consolidation to the first-line therapy [10]. Dialysis independence is a key to the improvement of the quality of life $[40,41]$. Thus, it should be considered as an ultimate goal after ASCT in patients with advanced CKD. Unfortunately, the majority of DD MM patients at ASCT already have irreversible kidney damage, and there is infrequent recovery of renal function. Kidney transplantation could be considered in MM patients in complete remission with standard risk disease, good functional capacity, and minimal comorbidities, but the issue of relapse and disease recurrence remains a barrier in the situation of high demand for renal allografts $[42,43]$.

In our study, we observed that DID MM patients had shorter PFS that DD MM (statistical comparison was not possible), but overall survival was similar in DD and DID patients. Future studies may address the impact of earlier dialysis initiation in patients with MM with advanced CKD and their qualification to ASCT.

It was reported that of patients with eGFR lower than $40 \mathrm{ml} / \mathrm{min}$ at diagnosis, more than half had reversal of their renal injury (RI) upon institution of anti-myeloma induction therapy; however, about $40 \%$ of patients did not demonstrate any reversal in renal function [44]. Furthermore, Gonsalves et al. demonstrated that even if a resolution of their RI upon receiving myeloma-directed therapy was achieved, the patients did not have equivalent survival outcomes to those of MM patients without RI. However, achievement of the reversal of RI was important for MM patients: patients who had RI at diagnosis but never recovered their renal function had a worse median OS when compared to patients who never had CKD (33 vs. 56 months, $p=0.006$ ) [44].

Among patients with CKD at diagnosis, there remains a substantial proportion for whom achievement of renal response is not possible even with the use of novel drugs. Management of these patients requires a multidisciplinary team approach including tailored treatment decisions depending on renal function, timing of initiation of dialysis, age, performance status, comorbidities, and consideration for ASCT. Thus, future treatment strategies should include efforts to improve renal function before transplant and qualification of patients to ASCT [12, 31, 32]. The study has several limitations. We analysed retrospectively 44 multiple myeloma patients with severe renal impairment, who underwent ASCT in the years 2004-2016 on behalf of the Polish Myeloma Study Group. This cohort of patients was a heterogeneous group of patients with MM treated with different induction regimens and transplanted at various time points from the diagnosis of their disease both in DD and NRF patients; therefore, we concluded that there were no clear guidelines on how to treat such patients. Because only two centres in Poland perform auto-HSCT in patients with end-stage renal disease (ESRD) and multiple myeloma, we had a chance to collect all Polish data. Patients did not undergo kidney biopsy as other reasons for progressive renal injury (for example: advanced diabetes mellitus with complications, advanced polycystic kidney disease) were excluded, and the reason for ESRD was advanced stage of multiple myeloma (with CRAB symptoms not only $R$ - renal). Cytogenetics and R-ISS might have helped to stratify risk in these patients, but these data were not available in a significant percentage of patients. The patients who were dialysis independent at diagnosis and became dialysis dependent due to disease progression were not included in the study because this group of patients have a different biology of multiple myeloma.

Prospective randomised studies in dialysis dependent patients may help to optimise the treatment of such patients to improve renal function and minimise treatment-related toxicity; however we are fully aware that enrollment for such a study might be a challenge as it was shown for the randomized controlled trials (RCTs) on the use 
of high cut-off membrane for cast nephrompathy in MM patients [46, 47].

In conclusion, optimal patient selection based upon good performance and higher albumin concentration (with every per increase of $1 \mathrm{~g} / \mathrm{dl}$ ), chemotherapy-responsive disease before ASCT, dose-adjusted melphalan, and aggressive postASCT supportive care are crucial to achieve acceptable results of treatment of MM patients with CKD, resulting in improved PFS and OS.

\section{Conflict of interest}

The authors declare no conflict of interest.

\section{References}

1. Knudsen LM, Nielsen B, Gimsing P, Geisler C. Autologous stem cell transplantation in multiple myeloma: outcome in patients with renal failure. Eur J Haematol 2005; 75: 27-33.

2. Matsue K, Fujiwara H, Iwama K, Kimura S, Yamakura M, Takeuchi M. Reversal of dialysis-dependent renal failure in patients with advanced multiple myeloma: single institutional experiences over 8 years. Ann Hematol 2010; 89: 291-7.

3. Knudsen LM, Hjorth M, Hippe E. Renal failure in multiple myeloma: reversibility and impact on the prognosis. Nordic Myeloma Study Group. Eur J Haematol 2000; 65: 175-81.

4. Badros A, Barlogie B, Siegel E, et al. Results of autologous stem cell transplant in multiple myeloma patients with renal failure. Br J Haematol 2001; 114: 822-9.

5. San Miguel JF, Lahuerta JJ, Garcia-Sanz R, et al. Are myeloma patients with renal failure candidates for autologous stem cell transplantation? Hematol J 2000; 1: 28-36.

6. St Bernard R, Chodirker L, Masih-Khan E, et al. Efficacy, toxicity and mortality of autologous SCT in multiple myeloma patients with dialysis-dependent renal failure. Bone Marrow Transplant 2015; 50: 95-9.

7. El Fakih R, Fox P, Popat U, et al. Autologous hematopoietic stem cell transplantation in dialysis-dependent myeloma patients. Clin Lymphoma Myeloma Leuk 2015; 15: 472-6.

8. Waszczuk-Gajda A, Drozd-Sokolowska J, Boguradzki P, et al. Stem cell mobilization in patients with dialysis-dependent multiple myeloma: Report of the Polish Myeloma Study Group. J Clin Apher 2018; 33: 249-58.

9. Waszczuk-Gajda A, Lewandowski Z, Drozd-Sokolowska J, et al. Autologous peripheral blood stem cell transplantation in dialysis-dependent multiple myeloma patients-DAUTOS Study of the Polish Myeloma Study Group. Eur J Haematol 2018; 101: 475-85.

10. Lee CK, Zangari M, Barlogie B, et al. Dialysis-dependent renal failure in patients with myeloma can be reversed by high-dose myeloablative therapy and autotransplant. Bone Marrow Transplant 2004; 33: 823-8.

11. Parikh GC, Amjad Al, Saliba RM, et al. Autologous hematopoietic stem cell transplantation may reverse renal failure in patients with multiple myeloma. Biol Blood Marrow Transplant 2009; 15: 812-6.

12. Augeul-Meunier K, Chretien ML, Stoppa AM, et al. Extending autologous transplantation as first line therapy in multiple myeloma patients with severe renal impair- ment: a retrospective study by the SFGM-TC. Bone Marrow Transplant 2018; 53: 749-55.

13. Dimopoulos MA, Sonneveld P, Leung N, et al. International Myeloma Working Group Recommendations for the diagnosis and management of myeloma-related renal impairment. J Clin Oncol 2016; 34: 1544-57.

14. Rajkumar SV. Multiple myeloma: 2016 update on diagnosis, risk-stratification, and management. Am J Hematol 2016; 91: 719-34.

15. Levey AS, Stevens LA, Schmid CH, et al. A new equation to estimate glomerular filtration rate. Ann Intern Med 2009; 150: 604-12.

16. Levey AS, Coresh J, Greene T, et al. Using standardized serum creatinine values in the modification of diet in renal disease study equation for estimating glomerular filtration rate. Ann Intern Med 2006; 145: 247-54.

17. Durie BG, Harousseau JL, Miguel JS, et al. International uniform response criteria for multiple myeloma. Leukemia 2006; 20: 1467-73.

18. https://www.eortc.be/services/doc/ctc/CTCAE_4.03_ 2010-0614_QuickReference_5x7.pdf.

19. Greipp PR, San Miguel J, Durie BG, et al. International staging system for multiple myeloma. J Clin Oncol 2005; 23: 3412-20.

20. Eleutherakis-Papaiakovou V, Bamias A, Gika D, et al. Renal failure in multiple myeloma: incidence, correlations, and prognostic significance. Leuk Lymphoma 2007; 48: 337-41.

21. Yang $M$, Zhang L, Wang X, Zhou Y, Wu S. Down-regulation of miR-203a by IncRNA PVT1 in multiple myeloma promotes cell proliferation. Arch Med Sci 2018; 14: 1333-9.

22. Breitkreutz I, Heiss C, Perne A, et al. Bortezomib improves outcome after SCT in multiple myeloma patients with end-stage renal failure. Bone Marrow Transplant 2014; 49: 1371-5.

23. Dimopoulos MA, Roussou M, Gkotzamanidou M, et al. The role of novel agents on the reversibility of renal impairment in newly diagnosed symptomatic patients with multiple myeloma. Leukemia 2013; 27: 423-9.

24. Dimopoulos MA, Terpos E, Chanan-Khan A, et al. Renal impairment in patients with multiple myeloma: a consensus statement on behalf of the International Myeloma Working Group. J Clin Oncol 2010; 28: 4976-84.

25. Attal M, Harousseau JL, Stoppa AM, et al. A prospective, randomized trial of autologous bone marrow transplantation and chemotherapy in multiple myeloma. Intergroupe Francais du Myelome. N Engl J Med 1996; 335: 91-7.

26. Tricot G, Alberts DS, Johnson C, et al. Safety of autotransplants with high-dose melphalan in renal failure: a pharmacokinetic and toxicity study. Clin Cancer Res 1996; 2: 947-52.

27. Nath CE, Trotman J, Tiley C, et al. High melphalan exposure is associated with improved overall survival in myeloma patients receiving high dose melphalan and autologous transplantation. Br J Clin Pharmacol 2016; 82: 149-59.

28. Tosi P, Zamagni E, Ronconi S, et al. Safety of autologous hematopoietic stem cell transplantation in patients with multiple myeloma and chronic renal failure. Leukemia 2000; 14: 1310-3.

29. Gera S, Musch E, Osterheld HK, Loos U. Relevance of the hydrolysis and protein binding of melphalan to the treatment of multiple myeloma. Cancer Chemother Pharmacol 1989; 23: 76-80.

30. Mahindra A, Hari P, Fraser R, et al. Autologous hematopoietic cell transplantation for multiple myeloma pa- 
tients with renal insufficiency: a center for international blood and marrow transplant research analysis. Bone Marrow Transplant 2017; 52: 1616-22.

31. Auner HW, lacobelli S, Sbianchi G, et al. Melphalan 140 $\mathrm{mg} / \mathrm{m}(2)$ or $200 \mathrm{mg} / \mathrm{m}(2)$ for autologous transplantation in myeloma: results from the Collaboration to Collect Autologous Transplant Outcomes in Lymphoma and Myeloma (CALM) study. A report by the EBMT Chronic Malignancies Working Party. Haematologica 2018; 103: 514-21.

32. Terpos E, Kleber M, Engelhardt M, et al. European Myeloma Network guidelines for the management of multiple myeloma-related complications. Haematologica 2015; 100: $1254-66$

33. Sweiss K, Patel S, Culos K, Oh A, Rondelli D, Patel P. Melphalan $200 \mathrm{mg} / \mathrm{m}$ (2) in patients with renal impairment is associated with increased short-term toxicity but improved response and longer treatment-free survival. Bone Marrow Transplant 2016; 51: 1337-41.

34. Carlson K. Melphalan $200 \mathrm{mg} / \mathrm{m} 2$ with blood stem cell support as first-line myeloma therapy: impact of glomerular filtration rate on engraftment, transplantation-related toxicity and survival. Bone Marrow Transplant 2005; 35: 985-90.

35. Alberts DS, Chen HG, Benz D, Mason NL. Effect of renal dysfunction in dogs on the disposition and marrow toxicity of melphalan. Br J Cancer 1981; 43: 330-4.

36. Shustik C, Bergsagel DE, Pruzanski W. Kappa and lambda light chain disease: survival rates and clinical manifestations. Blood 1976; 48: 41-51.

37. de Vries JC, Oortgiesen B, Hemmelder MH, et al. Restoration of renal function in patients with newly diagnosed multiple myeloma is not associated with improved survival: a population-based study. Leuk Lymphoma 2017; 58: 1-9.

38. Mavrakanas TA, Charytan DM. Cardiovascular complications in chronic dialysis patients. Curr Opin Nephrol Hypertens 2016; 25: 536-44.

39. Mostafa FA, Sad I, Elshamaa MF, et al. Left ventricular dysfunction by conventional and tissue Doppler echocardiography in pediatric hemodialysis patients: relation with plasma brain natriuretic peptide levels. Arch Med Sci Atheroscler Dis 2018; 3: e18-28.

40. Kusztal M, Trafidlo E, Madziarska K, et al. Depressive symptoms but not chronic pain have an impact on the survival of patients undergoing maintenance hemodialysis. Arch Med Sci 2018; 14: 265-75.

41. Sutkowski L, Matyja M, Pasternak A, Matyja A. WHOQOL-BREF survey of quality of life among dialyzed end-stage renal disease patients. Arch Med Sci Civil Dis 2018; 3: 112-20.

42. Van den Bosch I, Sprangers B, Gertz M. Multiple myeloma and kidney transplantation: the beginning of a new era. Clin Kidney J 2019; 12: 213-5.

43. Malyszko J, Dryl-Rydzynska T, Marcinkowski W, Prystacki T, Malyszko JS. Comorbidities on kidney transplantation waiting list relative to the status of the potential recipient. Arch Med Sci 2018; 14: 941-4.

44. Gonsalves WI, Leung N, Rajkumar SV, et al. Improvement in renal function and its impact on survival in patients with newly diagnosed multiple myeloma. Blood Cancer J 2015; 5: e296.

45. Dimopoulos MA, Roussou M, Gavriatopoulou M, et al. Bortezomib-based triplets are associated with a high probability of dialysis independence and rapid renal recovery in newly diagnosed myeloma patients with severe renal failure or those requiring dialysis. Am J Hematol 2016; 91: 499-502.

46. Bridoux F, Carron PL, Pegourie B, et al. Effect of high-cutoff hemodialysis vs conventional hemodialysis on hemodialysis independence among patients with myeloma cast nephropathy: a randomized clinical trial. JAMA 2017; 318: 2099-110.

47. Hutchison CA, Cockwell P, Moroz V, et al. High cutoff versus high-flux haemodialysis for myeloma cast nephropathy in patients receiving bortezomib-based chemotherapy (EULITE): a phase 2 randomised controlled trial. Lancet Haematol 2019; 6: e217-28. 